\title{
BIFURCATION IN THE MATHIEU EQUATION WITH THREE INDEPENDENT PARAMETERS*
}

\author{
BY
}

\author{
A. CAÑADA AND P. MARTINEZ-AMORES
}

Universidad de Granada

\section{Introduction. Consider the Mathieu equation}

$$
\ddot{x}+\left(\sigma^{2}+\epsilon \cos 2 t+\mu \cos 4 t\right) x=0
$$

where $\sigma^{2}, \epsilon, \mu$ are real parameters.

The basic problem is the following: find the values of $\sigma^{2}, \epsilon, \mu$ for which all solutions of (1.1) are stable. We shall call such values stable values, and unstable otherwise. Those parameter values which lie on surfaces bounding a stable zone are called transition points. They have the property that any neighborhood about them contains both stable and unstable points. A relatively simple method for obtaining the equations of the boundary surfaces of the stable zone (bifurcation surfaces) in the form $\sigma=\sigma(\epsilon, \mu)$ is based on the following observation: the question of stability of solutions depends on the Floquet multipliers $\lambda_{1}$ and $\lambda_{2}$ of (1.1). Moreover, these multipliers are continuous functions of $\sigma^{2}, \epsilon$, $\mu$. Thus, a point $\sigma^{2}, \epsilon, \mu$ for which one multiplier has absolute value greater than 1 cannot be a transition point: it must lie inside a zone of instability. Similarly, a point $\sigma^{2}, \epsilon, \mu$ for which the Floquet multipliers are complex conjugates of each other must lie inside a zone of stability. Consequently, the transition points correspond to values of $\sigma^{2}, \epsilon, \mu$ for which the Floquet multipliers equal \pm 1 .

From the Floquet theory for linear systems it follows that a periodic solution of period $\pi$ will exist for $\lambda_{1}=\lambda_{2}=1$ and that solutions of period $2 r$ will exist for $\lambda_{1}=\lambda_{2}=-1$. Thus, to calculate the bifurcation surfaces, i.e. those surfaces for which $\lambda= \pm 1$, we need only the surfaces on which there exist solutions of (1.1) with period $\pi$. For the case in which $\mu=$ 0 the zones of stability and instability are well known. For $\mu \neq 0$ this problem has been studied by Rubenfeld [4] by using a perturbation method in the theory of nonlinear oscillations. He obtains the bifurcation surfaces in the form of a series in terms of fractional powers of the parameters. In this work, we try to apply the method presented in $[1,2,3]$ to find the bifurcation surfaces of (1.1). This method is a special case of the method of Liapunov-Schmidt or the method of alternative problems. However, the form in which we obtain information from the alternative problem does not coincide with the classical approach. The reason for this is that the classical approach deals with problems with a single parameter, whereas our problem contains several parameters. The basic idea is to characterize the zeros of certain equations (bifurcation equations) in a neighborhood of zero by the sign of functionals. These functionals are related to the theory of universal unfoldings

\footnotetext{
* Received January 9, 1979. The authors would like to express their sincere thanks to Professor Jack K. Hale for his valuable comments and suggestions.
} 
of singularities of mappings of Thom (see [1]). The bifurcation surfaces are called catastrophe sets. However, Thom's theorem is not applicable to our special problem because the generic condition of this theorem is not satisfied. Nevertheless, we point out another way to study the zeros of the bifurcation equations by using the Weierstrass preparation theorem.

2. Mathematical preliminaries. Eq. (1.1) is equivalent to the system

$$
\dot{y}=A(t) y, \quad A(t)=\left[\begin{array}{cc}
0 & 1 \\
-\sigma^{2}-\epsilon \cos 2 t-\mu \cos 4 t & 0
\end{array}\right], y=\left[\begin{array}{l}
x \\
\dot{x}
\end{array}\right]=\left[\begin{array}{l}
y_{1} \\
y_{2}
\end{array}\right] .
$$

Suppose

$$
Y(t) \equiv\left[\begin{array}{cc}
x_{1}(t) & x_{2}(t) \\
\dot{x}_{1}(t) & \dot{x}_{2}(t)
\end{array}\right], \quad Y(0)=I,
$$

$x_{1}, x_{2}$ solutions of (1.1), is the principal matrix solution of (2.1) at $t=0$. The characteristic multipliers of (2.1) are the eigenvalues of the matrix $Y(\pi)$. Since $\operatorname{tr} A(t)=0$, the characteristic multipliers are the roots of the equation

$$
\lambda^{2}-2 B(\sigma, \epsilon, \mu)+1=0,
$$

where

$$
2 B(\sigma, \epsilon, \mu)=\operatorname{tr} Y(\pi)=x_{1}(\pi)+\dot{x}_{2}(\pi) .
$$

The function $B(\sigma, \epsilon, \mu)$ is continuous in the parameters $\sigma, \epsilon, \mu$ by the theorem of continuous dependence on parameters. For $\epsilon=0, \mu=0$, a principal matrix solution of (2.1) is

$$
Y(t)=\left[\begin{array}{cc}
\cos \sigma t & 1 / \sigma \sin \sigma t \\
-\sigma \sin \sigma t & \cos \sigma t
\end{array}\right]
$$

Therefore, $B(\sigma, 0,0) \neq \cos \sigma \pi$ and the characteristic multipliers are given by $\lambda=$ $B(\sigma, 0,0) \pm\left(B^{2}(\sigma, 0,0)-1\right)^{1 / 2}$. For $\sigma \neq n, n=0,1,2, \cdots$, we have $B^{2}(\sigma, 0,0)<1$. Note that $n=0$ corresponds to a constant, which is a solution of (1.1) when $\sigma=\epsilon=\mu=0$, and which may $B^{2}(\sigma, \epsilon, \mu)<1$, and therefore Eq. (1.1) is stable for $(\epsilon, \mu)$ in a neighborhood of $(0,0)$.

If $\sigma=n, n=1,2, \cdots$, then $B(\sigma, 0,0)= \pm 1$ and we cannot get any result by such an argument. Suppose now that we consider $\sigma$ as a parameter and try to determine $\sigma$ as a function of $(\epsilon, \mu)$ in such a way that $B(\sigma, \epsilon, \mu)= \pm 1$ for $\epsilon, \mu$ small. Then the characteristic multipliers are \pm 1 , and hence there is a periodic solution of periodic $\pi$ if $B(\sigma, \epsilon, \mu)=1(n$ even) or $2 \pi$ if $B(\sigma, \epsilon, \mu)=-1$ ( $n$ odd). As was said in the introduction, the surface $\sigma=\sigma(\epsilon$, $\mu), \sigma(0,0)=n$, will be the bifurcation surface desired.

At this point, we recall quickly the method of computing periodic solutions discussed in [3].

Let $\mathscr{P}_{\pi}=\left\{g: R \rightarrow R^{n}: g\right.$ is continuous, $\left.g(t+\pi)=g(t)\right\}$ and for any $g \in \mathscr{P}_{\pi}$, let $|g|=$ sup $\{|g(t)|, 0 \leq t \leq \pi\}$. If $g \in \mathscr{P}_{\pi}$ let $P: \mathscr{P}_{\pi} \rightarrow \mathscr{P}_{\pi}$ be the projection defined by the mean value of $g$; that is,

$$
P g=\frac{1}{\pi} \int_{0}^{\pi} g(t) d t
$$


Thus $P$ projects a function $g \in \mathscr{P}_{\pi}$ into a constant $n$ - vector. Now suppose $f: R \times R^{n} \times R^{k}$ $\rightarrow R^{n},(t, x, \lambda) \rightarrow f(t, x, \lambda)$, is continuous together with its first derivative with respect to $x$, $\lambda, f(t+\pi, x, \lambda)=f(t, x, \lambda), f(t, x, 0)=0$, and consider the equation

$$
\dot{x}=f(t, x, \lambda) \text {. }
$$

The linear equation is $\dot{x}=0$ and every solution of $\dot{x}=0$ is $\pi$-periodic, namely, constants. Then, Eq. (2.2) has a solution in $\mathscr{P}_{\pi}$ if and only if $P f(\cdot, x(\cdot), \lambda)=0$.

Since $P$ is a projection of $\mathscr{P}_{\pi}$, then

$$
\mathscr{P}_{\pi}=\mathscr{R}(P) \oplus \mathscr{N}(P)
$$

where $\mathscr{R}(P), \mathscr{N}(P)$ are the range and null space of $P$, respectively, and every element $f \in \mathscr{P}_{\pi}$ has the representation $f=P f+f-P f=P f+(I-P) f$. The operator $I-P$ is also a projection with range $\mathcal{M} P$ ). Elements $f$ of $\mathscr{R}(P)$ are characterized by the fact that $P f=f$.

Also, there is a continuous linear operator $K: \mathcal{N} P)=\mathscr{R}(I-P)=(I-P) \mathscr{P}_{\pi} \rightarrow$ $(I-P) \mathscr{P}_{\pi}$ such that $K(I-P) f(\cdot, x(\cdot), \lambda)$ is the unique solution of

$$
\dot{x}=(I-P) f(\cdot, x(\cdot), \lambda)
$$

which satisfies $P K(I-P) f=0$. Thus, $K$ is the unique primitive of $(I-P) f$ of mean value zero.

The general solution of $(2.3)$ is $\mathscr{P}_{\pi}$ is

$$
x=a+K(I-P) f(\cdot, x(\cdot), \lambda) .
$$

Hence, $x$ is a solution of (2.2) in $\mathscr{P}_{\pi}$ if and only if

$$
\begin{aligned}
& \text { (a) } x=a+y, y \in \mathscr{N}(P)=(I-P) \mathscr{P}_{\pi}, \\
& \text { (b) } y=K(I-P) f(\cdot, x(\cdot), \lambda), \\
& \text { (c) } 0=P f(\cdot, x(\cdot), \lambda),
\end{aligned}
$$

where $a$ is a constant $n$-vector such that $a=P x$. One can prove by a simple application of the contraction mapping principle (see Hale [3]) that for any $r$, there is a $\delta=\delta(r)$ and a unique solution $y^{*}(a, \lambda)$ of Eq. $(2.4 \mathrm{~b})$ for $|a|<r,|\lambda|<\delta, y^{*}(a, 0)=0$. Furthermore, every $\pi$-periodic solution of Eq. (2.2) can be obtained as $x^{*}=a+y^{*}(a, y)$ where $(a, \lambda)$ satisfy the bifurcation equations

$$
G(a, \lambda) \equiv P f\left(\cdot, a+y^{*}(a, \lambda), \lambda\right)=0 .
$$

Since the contraction mapping principle is used to obtain $y^{*}(a, \lambda)$, one can obtain the function $x^{*}=a+y^{*}$ by successive approximations:

$$
\begin{gathered}
x^{(0)}=a \\
x^{(k+1)}(t)=a+K(I-P) f\left(\cdot, x^{(k)}(\cdot), \lambda\right) \\
=a+\int(I-P) f\left(t, x^{(k)}(t), \lambda\right) d t, \quad k=0,1,2, \cdots,
\end{gathered}
$$

where $\int$ denotes the unique primitive of mean value zero.

It remains to discuss the bifurcation equation (2.5). There is one major difficulty in doing this and it is a characteristic property of perturbation problems which contain several independent small parameters. The function $G(a, 0)=0$ for all values of $a$. If the vec- 
tor parameters $\lambda=\left(\lambda_{1}, \cdots, \lambda_{k}\right)$ had the property that

$$
\lambda=\epsilon \mu, \mu=\left(\mu_{1}, \cdots, \mu_{k}\right), \quad \epsilon \in R,
$$

where the vector $\mu$ is fixed, then $G(a, \epsilon \mu)=\epsilon H(a, \epsilon)$ and, for $\epsilon \neq 0$, the bifurcation equations (2.5) would be equivalent to

$$
H(a, \epsilon)=0 .
$$

It is now possible that $H(a, \epsilon) \equiv 0$ for all values of $a$. One can determine the values of $a, \epsilon$ for which $H(a, \epsilon)=0$ and obtain $\pi$-periodic solution of Eq. (2.2) for $\epsilon$ small, $|\epsilon|<\epsilon_{0}$. Of course, the value of $\epsilon_{0}$ will depend in general upon the particular vector $\mu, \epsilon_{0}=\epsilon_{0}(\mu)$. To be sure that a full neighborhood of $\lambda=0$ is discussed, one must take all possible directions $\mu$ and have a procedure for showing that there is an $\epsilon_{0}{ }^{*}>0$ such that $\epsilon_{0}(\mu) \geq \epsilon_{0}{ }^{*}$ for all directions $\mu$. Classical perturbation procedures do not give such lower bounds on $\epsilon_{0}(\mu)$. Below, we give a procedure for doing this for Mathieu's equation.

Let us suppose $f(t, x, \lambda)$ is linear in $x$; that is, consider the equation

$$
\dot{x}(t)=A(t, \lambda) x, \quad A(t, 0)=0
$$

where $A(t+\pi, \lambda)=A(t, \lambda)$ is an $n \times n$ matrix. We apply the above procedure to obtain $\pi$ periodic solutions of Eq. (2.7). In this case, it is very easy to observe from the uniqueness of the function $y^{*}(a, \lambda)$ that $y^{*}(a, \lambda)$ is linear in $a$. Thus, $x^{*}(a, \lambda)=a+y^{*}(a, \lambda)$ is linear in $a$,

$$
x^{*}(a, \lambda)=a+y^{*}(a, \lambda)=B(\lambda) a, \quad B(0)=I,
$$

where $B(\lambda)$ is an $n \times n$ matrix function of $\lambda$. Consequently, using (2.7), (2.8) the bifurcation equations (2.5) are equivalent to

$$
\left[\frac{1}{\pi} \int_{0}^{\pi} A(t, \lambda) B(\lambda) d t\right] a=0 .
$$

If the function $x^{*}(a, \lambda)$ is to be a nontrivial $\pi$-periodic solution of Eq. (2.7), then we must have $a \neq 0$. This is possible if and only if $\lambda$ satisfies the scalar equation

$$
\operatorname{det} D(\lambda)=0, \quad D(\lambda)=\frac{1}{\pi} \int_{0}^{\pi} A(t, \lambda) B(\lambda) d t .
$$

We have thus reduced the question of the existence of $\pi$-periodic solutions of the linear system (2.7) to the determination of the solutions $\lambda$ of Eq. (2.10) near $\lambda=0$. A complete discussion of Eq. (2.10) is extremely difficult. In the next section, we discuss it for Mathieu's equation.

We remark that if Eq. (2.7) is an analytic function of $\lambda$ near $\lambda=0$, then det $D(\lambda)$ is an analytic function of $\lambda$ near $\lambda=0$.

3. The Mathieu equation. To apply the results of the previous section to (2.1), we use the transformation of van der Pol,

$$
\begin{aligned}
& y_{1}=z_{1} \sin n t+z_{2} \cos n t \\
& y_{2}=n\left(z_{1} \cos n t-z_{2} \sin n t\right),
\end{aligned}
$$


for any given integer $n>0$. This transformation applied to (2.1) yields the equivalent system

$$
\begin{aligned}
& \dot{z}_{1}=\frac{1}{n}\left(n^{2}-\sigma^{2}-\epsilon \cos 2 t-\mu \cos 4 t\right)\left(z_{1} \sin n t+z_{2} \cos n t\right) \cos n t \\
& \dot{z}_{2}=-\frac{1}{n}\left(n^{2}-\sigma^{2}-\epsilon \cos 2 t-\mu \cos 4 t\right)\left(z_{1} \sin n t+z_{2} \cos n t\right) \sin n t
\end{aligned}
$$

which is a special case of system (2.7). Before applying the results of Sec. 2, let us write (3.1) in vector form. After some laborious computations, we obtain

$$
\begin{aligned}
\dot{z}= & \frac{1}{2 n}\left[\alpha A \sin 2 n t+\alpha B \cos 2 n t+\alpha C-\frac{\epsilon}{2} A \sin (2 n+2) t\right. \\
& -\frac{\epsilon}{2} A \sin (2 n-2) t-\epsilon C \cos 2 t-\frac{\epsilon}{2} B \cos (2 n+2) t \\
& -\frac{\epsilon}{2} B \cos (2 n-2) t-\frac{\mu}{2} A \sin (2 n+4) t-\frac{\mu}{2} A \sin (2 n-4) t \\
& \left.-\mu C \cos 4 t-\frac{\mu}{2} B \cos (2 n+4) t-\frac{\mu}{2} B \cos (2 n-4) t\right] z \\
\equiv & \frac{1}{2 n} Q(t) z \equiv A(t, \alpha, \epsilon, \mu) z
\end{aligned}
$$

where

$$
\begin{gathered}
z=\operatorname{col}\left(z_{1}, z_{2}\right) \alpha=n^{2}-\sigma^{2} \\
A=\left[\begin{array}{cc}
1 & 0 \\
0 & -1
\end{array}\right], \quad B=\left[\begin{array}{ll}
0 & 1 \\
1 & 0
\end{array}\right], \quad C=\left[\begin{array}{cc}
0 & 1 \\
-1 & 0
\end{array}\right] .
\end{gathered}
$$

If $n=1$ and we apply (2.5) with $z^{(0)}=\operatorname{col}\left(a_{1}, a_{2}\right)$, then it is easy to see that the matrix $D(\lambda)$ in Eq. (2.10), $\lambda=(\alpha, \epsilon, \mu)$, satisfies

$$
D(\alpha, \epsilon, \mu)=\alpha C-\frac{\epsilon}{2} B+O\left((|\alpha|+|\epsilon|)^{2}+(|\alpha|+|\epsilon|)|\mu|\right)
$$

as $\alpha, \epsilon, \mu \rightarrow 0$. Consequently, the bifurcation equation becomes

$$
\operatorname{det} D(\alpha, \epsilon, \mu)=\left(\alpha-\frac{\epsilon}{2}\right)\left(\alpha+\frac{\epsilon}{2}\right)+O\left((|\alpha|+|\epsilon|)^{2}(|\alpha|+|\epsilon|+|\mu|)\right)=0
$$

as $\alpha, \epsilon, \mu \rightarrow 0$. It is intuitively clear that this equation has only two solutions of the form $\alpha= \pm \epsilon \backslash 2+O\left(|\epsilon|^{2}(1+|\mu|)\right)$ as $\epsilon, \mu \rightarrow 0$ near $(\alpha, \epsilon, \mu)=(0,0,0)$. However, the implicit function theorem is not applicable here. To prove the solutions have this form, we first make the observation that there is a $k>0$ and a neighborhood $V$ of $(\alpha, \epsilon)=,(0,0,0)$ such that every solution of Eq. (3.3) in $V$ satisfies

$$
|\alpha| \leq k|\epsilon| .
$$


In fact, if this is not so there is a sequence of solutions $\left(\alpha_{j}, \epsilon_{j}, \mu_{j}\right) \rightarrow(0,0,0), \epsilon_{j} / \alpha_{j} \rightarrow 0$ as $j \rightarrow \infty, \alpha_{j} \neq 0, j=1,2, \cdots$, . Then

$$
0=\frac{\operatorname{det} D\left(\alpha_{j}, \epsilon_{j}, \mu_{j}\right)}{\alpha_{j}^{2}} \rightarrow 1 \quad \text { as } j \rightarrow \infty,
$$

which is a contradiction. Therefore, relation (3.4) is valid.

From (3.4), we are justified in scaling $\alpha=\epsilon \beta$ to obtain the equivalent equations

$$
D_{1}(\beta, \epsilon, \mu) \equiv \frac{\operatorname{det} D(\epsilon \beta, \epsilon, \mu)}{\epsilon^{2}}=\left(\beta-\frac{1}{2}\right)\left(\beta+\frac{1}{2}\right)+O(|\epsilon|+|\mu|)=0 .
$$

One can now apply the implicit function theorem to $D_{1}(\beta, \epsilon, \mu)$ to obtain the two unique solutions $\beta= \pm 1 / 2+O(|\epsilon|+|\mu|)$ in a neighborhood of $(\epsilon, \mu)=(0,0)$. This gives $\alpha=\epsilon \beta$, as desired. Thus

$$
\sigma^{2}=1 \pm \epsilon / 2+O\left(|\epsilon|^{2}(1+|\mu|)\right)
$$

as $(\epsilon, \mu) \rightarrow(0,0)$.

For $n=2$, the same procedure yields

$$
D(\alpha, \epsilon, \mu)=\alpha C-\frac{\mu}{2} B+O\left((|\alpha|+|\mu|)^{2}+(|\alpha|+|\mu|)|\epsilon|\right)
$$

as $\alpha, \epsilon, \mu \rightarrow 0$. The same procedure gives

$$
\sigma^{2}=4 \pm \mu / 2+O\left(|\mu|^{2}(1+|\epsilon|)\right)
$$

as $(\epsilon, \mu) \rightarrow(0,0)$.

For $n=3$, the first approximation to $D(\alpha, \epsilon, \mu)$ is $I$ and no information is obtained about the bifurcation surfaces. So, to obtain the bifurcation surfaces for $n=3$ one must go to higher approximations until the two surfaces $\sigma=\sigma(\epsilon, \mu)$ become different. To do this we use (2.6). Then

$$
\begin{aligned}
z^{(1)}= & z^{(0)}+\int(I-P) A(t, \alpha, \epsilon, \mu) z^{(0)} d t \\
= & z^{(0)}+\int\left(A(t, \alpha, \epsilon, \mu)-\frac{\alpha C}{2 n}\right) z^{(0)} d t \\
= & z^{(0)}+\frac{1}{2 n}\left[-\frac{\alpha A}{2 n} \cos 2 n t+\frac{\alpha B}{2 n} \sin 2 n t+\frac{\epsilon A}{2(2 n+2)} \cos (2 n+2) t\right. \\
& +\frac{\epsilon A}{2(2 n-2)} \cos (2 n-2) t-\frac{\epsilon C}{2} \sin 2 t-\frac{\epsilon B}{2(2 n+2)} \sin (2 n+2) t \\
& -\frac{\epsilon B}{2(2 n-2)} \sin (2 n-2) t+\frac{\mu A}{2(2 n+4)} \cos (2 n+4) t+\frac{\mu A}{2(2 n-4)} \cos (2 n-4) t \\
& \left.-\frac{\mu C}{4} \sin 4 t-\frac{\mu B}{2(2 n+4)} \sin (2 n+4) t-\frac{\mu B}{2(2 n-4)} \sin (2 n-4) t\right] z^{(0)} \\
& \equiv z^{(0)}+\frac{1}{2 n} R(t) z^{(0)} .
\end{aligned}
$$


Since

$$
A(t, \alpha, \epsilon, \mu) z^{(1)}=\frac{1}{2 n} Q(t) z^{(1)}=\left[\frac{1}{2 n} Q(t)+\frac{1}{4 n^{2}} Q(t) R(t)\right] z^{(0)},
$$

if we apply (2.5) for $n=3$, we will have the second approximation for the bifurcation equations. After tedious computations we get that

$$
\begin{aligned}
P A(., \alpha, \epsilon, \mu) z^{(1)}= & \frac{1}{6} \alpha C z^{(0)}+\frac{1}{36}\left[\frac{\alpha^{2} A B}{12}-\frac{\alpha^{2} B A}{12}+\epsilon^{2} A B+\frac{\epsilon^{2} A B}{32}\right. \\
& +\frac{\epsilon \mu A C}{16}-\frac{\epsilon \mu C A}{8}-\frac{\epsilon^{2} B A}{64}-\frac{\epsilon^{2} B A}{32}+\frac{\mu^{2} A B}{80} \\
& +\frac{\mu \epsilon A C}{8}+\frac{\mu^{2} A B}{16} \\
& \left.-\frac{\mu \epsilon C A}{16}-\frac{\mu^{2} B A}{80}-\frac{\mu^{2} B A}{16}\right] z^{(0)} \\
& =\left[\left(\frac{\alpha}{6}+\frac{\alpha^{2}}{216}+\frac{\epsilon^{2}}{384}+\frac{\mu^{2}}{240}\right) C+\frac{\mu \epsilon}{96} B\right] z^{(0)}
\end{aligned}
$$

since $A B=C, A C=B, B A=-C, C A=-B$. If we continue with higher approximations, it is clear that the matrix $D$ in Eq. (2.10) satisfies

$$
D(\alpha, \epsilon, \mu)=\left(\frac{\alpha}{6}+\frac{\epsilon^{2}}{384}+\frac{\mu^{2}}{240}\right) C+\frac{\mu \epsilon}{96} B+O\left(|\alpha|(|\alpha|+|\epsilon|+|\mu|)+(|\epsilon|+|\mu|)^{3}\right)
$$

as $(\alpha, \epsilon, \mu) \rightarrow(0,0,0)$. Consequently, the bifurcation equations are

$$
\begin{aligned}
36 \operatorname{det} D(\alpha, \epsilon, \mu)= & \left(\alpha+\frac{\epsilon^{2}}{64}+\frac{\mu^{2}}{40}+\frac{\epsilon \mu}{16}\right)\left(\alpha+\frac{\epsilon^{2}}{64}+\frac{\mu^{2}}{40}-\frac{\epsilon \mu}{16}\right) \\
& +O\left(\left[(|\epsilon|+|\mu|)^{2}+|\alpha|\right]^{2}\left(|\epsilon|+|\mu|+|\alpha|^{2}\right)\right)=0
\end{aligned}
$$

as $(\alpha, \epsilon, \mu) \rightarrow(0,0,0)$. As before, one shows that any small solution of $D(\alpha, \epsilon, \mu)=0$ has the a priori bound

$$
|\alpha| \leq k(|\epsilon|+|\mu|)^{2}
$$

as $\epsilon, \mu \rightarrow 0$. This justifies the scaling

$$
\alpha=\epsilon^{2} \beta, \quad \mu=\epsilon \nu
$$

to obtain the equivalent equation

$$
\left(\beta+\frac{1}{64}+\frac{\nu^{2}}{40}+\frac{\nu}{16}\right)\left(\beta+\frac{1}{64}+\frac{\nu^{2}}{40}-\frac{\nu}{16}\right)+O(|\epsilon|)=0
$$

as $|\epsilon| \rightarrow 0$. We must consider solutions of this equation for $\beta \in R, \mu \in R$ and $|\epsilon|$ small.

For simplicity in discussing this equation, let

$$
\beta+\frac{1}{64}=\gamma
$$


The equation becomes

$$
h(\gamma, \nu, \epsilon) \equiv\left(\gamma+\frac{\nu^{2}}{40}+\frac{\nu}{16}\right)\left(\gamma+\frac{\nu^{2}}{40}-\frac{\nu^{2}}{16}\right)+O(|\epsilon|)=0 .
$$

For $\epsilon=0, \nu=0$, this equation has a double zero at $\gamma=0$ and $h(\gamma, \nu, \epsilon)$ has a minimum at $\nu=0, \epsilon=0$. Since

$$
\frac{\partial^{2} h}{\partial \gamma^{2}}(0,0,0)=2 \neq 0
$$

there is a unique function $\gamma^{*}(\nu, \epsilon), \gamma^{*}(0,0)=0$, such that

$$
\frac{\partial h}{\partial \gamma}\left(\gamma^{*}(\nu, \epsilon), \nu, \epsilon\right)=0
$$

for $(\nu, \epsilon)$ in a neighborhood of zero. The function $h$ has a minimum at $\gamma^{*}(\nu, \epsilon)$. Define the functional

$$
m(\nu, \epsilon)=h\left(\gamma^{*}(\nu, \epsilon), \nu, \epsilon\right) .
$$

If $m(\nu, \epsilon)>0$, there are no solutions of the bifurcation equations; if $m(\nu, \epsilon)<0$ there are two distinct solutions of the bifurcation equations (see [1]).

It follows from (3.7) that $\gamma^{*}=-\left(v^{2} / 40\right)$ and that

$$
m(\nu, \epsilon)=-\frac{\nu^{2}}{16^{2}}+O(|\epsilon|) .
$$

To decide if $m(\nu, \epsilon)$ changes sign for $(\nu, \epsilon)$ in a neighborhood of zero we return to equation and we get next approximation for the terms $\epsilon^{3}$ in $D(\alpha, \epsilon, \mu)$. Again, to do this we use (2.6). Then

$$
z^{(2)}=z^{(0)}+\int(I-P) A(t, \alpha, \epsilon, \mu) z^{(1)} d t=z^{(1)}+\frac{1}{36} \int Q(t) R(t) z^{(0)} d t
$$

after taking into account $z^{(1)}$ and the definitions of $Q(t)$ and $R(t)$.

If these computations are done, we compute $A(t, \alpha, \epsilon, \mu) z^{(2)}$ and then we apply (2.5); we get that

$$
\begin{aligned}
P A(., \alpha, \epsilon, \mu) z^{(2)}= & \left\{\left[\frac{\mu \epsilon}{96}+\frac{1}{432}\left(\frac{-\epsilon^{3}}{64}+\frac{127 \alpha \epsilon \mu}{192}\right)\right] B\right. \\
& \left.+\left[\frac{\alpha}{6}+\frac{\alpha^{2}}{216}+\frac{\epsilon^{2}}{384}+\frac{\mu^{2}}{240}+\frac{1}{432}\left(\frac{\alpha^{3}}{18}+\frac{45 \alpha \epsilon^{2}}{128}+\frac{33 \alpha \mu^{2}}{100}-\frac{17 \epsilon^{2} \mu}{160}\right)\right] C\right\} z^{(0)} .
\end{aligned}
$$

Hence the matrix $D$ in (2.10) satisfies

$$
\begin{aligned}
D(\alpha, \epsilon, \mu)= & {\left[\frac{\mu \epsilon}{96}+\frac{1}{432}\left(\frac{-\epsilon^{3}}{64}+\frac{127 \alpha \epsilon \mu}{192}\right)\right] B+\left[\frac{\alpha}{6}+\frac{\epsilon^{2}}{384}+\frac{\mu^{2}}{240}\right.} \\
& \left.+\frac{1}{432}\left(\frac{45 \alpha \epsilon^{2}}{128}+\frac{33 \alpha \mu^{2}}{100}-\frac{17 \epsilon^{2} \mu}{160}\right)\right] C+\text { h.o.t. }
\end{aligned}
$$

where h.o.t. designates terms of higher order of the form

$$
\text { h.o.t. } \equiv O\left(|\alpha|(|\alpha|+|\epsilon|+|\mu|)+(|\epsilon|+|\mu|)\left(|\epsilon|^{3}+|\mu|^{2}\right)\right) .
$$


Consequently, the bifurcation equations are

$$
\begin{aligned}
36 \operatorname{det} D(\alpha, \epsilon, \mu)= & \left(\alpha+\frac{\epsilon^{2}}{64}+\frac{\mu^{2}}{40}+\frac{\epsilon \mu}{16}\right)\left(\alpha+\frac{\epsilon^{2}}{64}+\frac{\mu^{2}}{40}-\frac{\epsilon \mu}{16}\right) \\
& +\frac{1}{72}\left(\alpha+\frac{\epsilon^{2}}{64}+\frac{\mu^{2}}{40}-\frac{\epsilon \mu}{16}\right)\left(\frac{-\epsilon^{3}}{64}+\frac{127 \alpha \epsilon \mu}{192}+\frac{45 \alpha \epsilon^{2}}{128}+\frac{33 \alpha \mu^{2}}{100}-\frac{17 \epsilon^{2} \mu}{160}\right) \\
& -\frac{1}{72}\left(\alpha+\frac{\epsilon^{2}}{64}+\frac{\mu^{2}}{40}+\frac{\epsilon \mu}{16}\right)\left(\frac{-\epsilon^{3}}{64}+\frac{127 \alpha \epsilon \mu}{192}-\frac{45 \alpha \epsilon^{2}}{128}-\frac{33 \alpha \mu^{2}}{100}+\frac{17 \mu \epsilon^{2}}{160}\right) \\
& +\frac{1}{72^{2}}\left(\frac{-\epsilon^{3}}{64}+\frac{127 \alpha \epsilon \mu}{192}+\frac{45 \alpha \epsilon^{2}}{128}+\frac{33 \alpha \mu^{2}}{100}-\frac{17 \epsilon^{2} \mu}{160}\right) \\
& \cdot\left(\frac{-\epsilon^{3}}{64}+\frac{127 \alpha \epsilon \mu}{192}-\frac{45 \alpha \epsilon^{2}}{128}-\frac{33 \alpha \mu^{2}}{100}+\frac{17 \epsilon^{2} \mu}{160}\right) \\
& + \text { h.o.t. }=0, \text { as }(\alpha, \epsilon, \mu) \rightarrow(0,0,0) .
\end{aligned}
$$

By using the scaling (3.6) we obtain the equivalent equation

$36 \operatorname{det} D(\beta, \nu, \epsilon)=\left(\beta+\frac{1}{64}+\frac{\nu^{2}}{40}+\frac{\nu}{16}\right)\left(\beta+\frac{1}{64}+\frac{\nu^{2}}{40}-\frac{\nu}{16}\right)$

$$
\begin{aligned}
& +\frac{1}{72}\left(\beta+\frac{1}{64}+\frac{\nu^{2}}{40}-\frac{\nu}{16}\right)\left(\frac{-\epsilon}{64}+\frac{127 \beta \epsilon^{2} \nu}{192}+\frac{45 \epsilon^{2} \beta}{128}+\frac{33 \beta \epsilon^{2} \nu^{2}}{100}-\frac{17 \epsilon \nu}{160}\right) \\
& -\frac{1}{72}\left(\beta+\frac{1}{64}+\frac{\nu^{2}}{40}+\frac{\nu}{16}\right)\left(\frac{-\epsilon}{64}+\frac{127 \epsilon^{2} \beta \nu}{192}-\frac{45 \epsilon^{2} \beta}{128}-\frac{33 \beta \epsilon^{2} \nu^{2}}{100}+\frac{17 \epsilon \nu}{160}\right) \\
& -\frac{1}{72}\left(\frac{-\epsilon}{64}+\frac{127 \epsilon^{2} \nu \beta}{192}+\frac{45 \epsilon^{2} \beta}{128}+\frac{33 \beta \epsilon^{2} \nu^{2}}{100}-\frac{17 \epsilon \nu}{160}\right)\left(-\frac{\epsilon}{64}+\frac{127 \epsilon^{2} \nu \beta}{192}\right. \\
& \left.-\frac{45 \epsilon^{2} \beta}{128}-\frac{33 \beta \epsilon^{2} \nu^{2}}{100}+\frac{17 \epsilon \nu}{160}\right)+O\left(|\epsilon|^{4}\right)=0,
\end{aligned}
$$

as $|\epsilon| \rightarrow 0$. Since we are interested only in the terms $\epsilon^{3}$ in $D(\alpha, \epsilon, \mu)$ or, equivalently, in the terms $\epsilon$ in $D(\beta, \nu, \epsilon)$, we can write the bifurcation equation as

$$
\begin{aligned}
& \left(\beta+\frac{1}{64}+\frac{\nu^{2}}{40}+\frac{\nu}{16}\right)\left(\beta+\frac{1}{64}+\frac{\nu^{2}}{40}-\frac{\nu}{16}\right)+\frac{1}{72}\left(\beta+\frac{1}{64}+\frac{\nu^{2}}{40}-\frac{\nu}{16}\right)\left(\frac{-\epsilon}{64}-\frac{17 \epsilon \nu}{160}\right) \\
& -\frac{1}{72}\left(\beta+\frac{1}{64}+\frac{\nu^{2}}{40}+\frac{\nu}{16}\right)\left(\frac{-\epsilon}{64}+\frac{17 \epsilon \nu}{160}\right)=\left(\beta+\frac{1}{64}+\frac{\nu^{2}}{40}+\frac{\nu}{16}\right)\left(\beta+\frac{1}{64}+\frac{\nu^{2}}{40}-\frac{\nu}{16}\right) \\
& -\frac{2}{72}\left(\beta+\frac{1}{64}+\frac{\nu^{2}}{40}\right) \frac{17 \epsilon \nu}{160}+\frac{2 \epsilon \nu}{72 \cdot 16 \cdot 64}+O\left(|\epsilon|^{2}\right)=0 .
\end{aligned}
$$

Let $\beta+(1 / 64)=\gamma$. This equation becomes

$h(\gamma, \epsilon, \nu) \equiv\left(\gamma+\frac{\nu^{2}}{40}+\frac{\nu}{16}\right)\left(\gamma+\frac{\nu^{2}}{40}-\frac{\nu}{16}\right)-\frac{2}{72}\left(\gamma+\frac{\nu^{2}}{40}\right) \frac{17 \epsilon \nu}{160}+\frac{2 \epsilon \nu}{72 \cdot 16 \cdot 64}+O\left(|\epsilon|^{2}\right)=0$ as $|\epsilon| \rightarrow 0$.

Substituting $\gamma^{*}=\left(-\nu^{2} / 40\right)$ in this equation, we get that

$$
m(\nu, \epsilon)=-\frac{\nu^{2}}{16^{2}}+\frac{\epsilon \nu}{36 \cdot 16 \cdot 64}+O\left(|\epsilon|^{2}\right)=\frac{1}{16^{2}}\left(-\nu^{2}+\frac{\nu \epsilon}{144}\right)+O\left(|\epsilon|^{2}\right)
$$


The term $-\nu^{2}+(\nu \epsilon / 144)$ represents an indefinite quadratic form. Therefore, neglecting the higher-order terms in (3.9), we have that $m(\nu, \epsilon)$ changes sign for $(\nu, \epsilon)$ near $(0,0)$ and then Eq. (3.7) has two unique solutions; that is, the bifurcation values $\gamma$ are

$$
\gamma=\frac{\nu^{2}}{40} \pm \frac{\nu}{16}+O\left(|\epsilon|(1+|\nu|)+|\nu|^{3}\right)
$$

for $(\nu, \epsilon)$ near $(0,0)$.

The above analysis completes the discussion for $|\nu||<\delta,| \epsilon \mid<\epsilon_{0}, \delta>0, \epsilon_{0}>0$. if $\delta \leq$ $|\nu| \leq \delta_{1}$, where $\delta_{1}$ is any fixed number, then $h(\gamma, \nu, 0)=0$ has only simple zeros. Consequently, the implicit function theorem implies we can choose $\epsilon_{0}>0$ sufficiently small that the zeros of $h(\gamma, \nu, \epsilon)$ are determined for $|\epsilon|<\epsilon_{0}, \delta \leq|\nu| \leq \delta_{1}$.

To handle the case where $\nu$ is very large, let us use the scaling

$$
\alpha=\mu^{2} \beta, \quad \epsilon=\mu \bar{\epsilon}
$$

in Eq. (3.5) to obtain the equivalent equation

$$
\left(\beta+\frac{\bar{\epsilon}^{2}}{64}+\frac{1}{40}+\frac{\bar{\epsilon}}{16}\right)\left(\beta+\frac{\bar{\epsilon}^{2}}{64}+\frac{1}{40}-\frac{\bar{\epsilon}}{16}\right)+O(|\mu|)=0 .
$$

We are interested in this equation for $(\bar{\epsilon}, \mu)$ near $(0,0)$, since $\bar{\epsilon}=0$ corresponds to $\delta_{1}=$ $\infty$.

Let $\beta+(1 / 40)=\bar{\gamma}$. Then this equation becomes

$$
\bar{h}(\bar{\gamma}, \bar{\epsilon}, \mu) \equiv\left(\bar{\gamma}+\frac{\bar{\epsilon}^{2}}{64}+\frac{\bar{\epsilon}}{16}\right)\left(\bar{\gamma}+\frac{\bar{\epsilon}^{2}}{64}-\frac{\bar{\epsilon}}{16}\right)+O(|\mu|)=0 .
$$

Again, we have a double root for $(\bar{\epsilon}, \mu)=(0,0)$. The function $\bar{h}$ has a minimum at $\bar{\gamma}^{*}=$ $\left(-\bar{\epsilon}^{2} / 64\right)$. Then

$$
\bar{m}(\bar{\epsilon}, \mu)=\bar{h}\left(\bar{\gamma}^{*}(\bar{\epsilon}, \mu), \bar{\epsilon}, \mu\right)=-\frac{\bar{\epsilon}^{2}}{16^{2}}+O(|\mu|) .
$$

In a manner similar to the one before, to decide if $\bar{m}(\bar{\epsilon}, \mu)$ changes sign for $(\bar{\epsilon}, \mu)$ near $(0,0)$ we get a next approximation for the terms $\epsilon^{3}$ in $D(\alpha, \epsilon, \mu)$. We get that

$$
\bar{h}(\bar{\gamma}, \bar{\epsilon}, \mu)=\left(\bar{\gamma}+\frac{\bar{\epsilon}^{2}}{64}+\frac{\bar{\epsilon}}{16}\right)\left(\bar{\gamma}+\frac{\bar{\epsilon}^{2}}{64}-\frac{\bar{\epsilon}}{16}\right)-\frac{2}{72}\left(\bar{\gamma}+\frac{\bar{\epsilon}}{64}\right) \frac{17 \mu \bar{\epsilon}^{2}}{160}+\frac{2 \mu \bar{\epsilon}^{4}}{72 \cdot 16 \cdot 64}+O\left(|\mu|^{2}\right)
$$

and that

$$
\bar{m}(\bar{\epsilon}, \mu)=-\frac{\bar{\epsilon}^{2}}{16^{2}}+\frac{\mu \bar{\epsilon}^{4}}{36 \cdot 16 \cdot 64}+O\left(|\mu|^{2}\right)=\frac{\bar{\epsilon}^{2}}{16^{2}}\left(-1+\frac{\mu \bar{\epsilon}^{2}}{144}\right)+O\left(|\mu|^{2}\right) .
$$

Therefore, up to higher-order terms in $\bar{\epsilon}, \mu, \bar{m}(\bar{\epsilon}, \mu)$ changes sign for $(\bar{\epsilon}, \mu)$ near $(0,0)$, and thus (3.10) has two unique solutions for $(\bar{\epsilon}, \mu)$ in a neighborhood of zero. This completes the argument for $\nu$ large.

Thus, for $n=3$, the bifurcation surfaces are

$$
\sigma^{2}=9+\frac{\epsilon^{2}}{64}+\frac{\mu^{2}}{40} \pm \frac{\epsilon \mu}{16}+O\left((|\epsilon|+|\mu|)^{3}\right),
$$

as $(\epsilon, \mu) \rightarrow(0,0)$.

We could have proceeded to analyze the bifurcation equations (3.5) by applying the 
Weierstrass preparation theorem for analytic functions (see [5]). In (3.5), define $f(\alpha, \epsilon, \mu)=\left(\alpha+\frac{\epsilon^{2}}{64}+\frac{\mu^{2}}{40}+\frac{\epsilon \mu}{16}\right)\left(\alpha+\frac{\epsilon^{2}}{64}+\frac{\mu^{2}}{40}-\frac{\epsilon \mu}{16}\right)+O\left(\left[(|\epsilon|+|\mu|)^{2}+|\alpha|\right]^{2}\left(|\epsilon|+|\mu|+|\alpha|^{2}\right)\right)$.

Since $f(0,0,0)=0$ and $f(\alpha, 0,0)=\alpha^{2}+O\left(|\alpha|^{4}\right)$, the Weierstrass preparation theorem implies there is a polynomial $P_{2}(\alpha, \epsilon, \mu)=\alpha^{2}+a \alpha+b$, where $a, b$ are analytic in $\epsilon$, $\mu$, and an analytic function $q(\alpha, \epsilon, \mu)$ such that $q(\alpha, \epsilon, \mu) \neq 0$ in a neighborhood of $(0,0,0)$, and

$$
f(\alpha, \epsilon, \mu)=p_{2}(\alpha, \epsilon, \mu) q(\alpha, \epsilon, \mu) .
$$

Furthermore, the coefficients $a, b$ in $p_{2}$ are computable in terms of $\epsilon, \mu$.

If we let $\alpha=\bar{\alpha}-(a / 2), \lambda=b-\left(a^{2} / 4\right)$, and $\bar{p}_{2}(\bar{\alpha}, \lambda)=\bar{\alpha}^{2}+\lambda$, then the number of zeros of $\bar{p}_{2}(\bar{\alpha}, \lambda)$ coincides with the number of zeros of $f(\alpha, \epsilon, \mu)$. If $\lambda>0$, there are no zeros, if $\lambda=0$, there is one double zero and, if $\lambda<0$, there are two simple zeros. Since

$$
f(\alpha, \epsilon, \mu)=\alpha^{2}+\left(\frac{\epsilon^{2}}{32}+\frac{\mu^{2}}{20}\right)+\frac{\epsilon^{4}}{64^{2}}+\frac{\mu^{4}}{40^{2}}-\frac{\epsilon^{2} \mu^{2}}{320}+O\left(\left[(|\epsilon|+|\mu|)^{2}+|\alpha|\right]^{2}\left(|\epsilon|+|\mu|+|\alpha|^{2}\right)\right]
$$

if we consider only up to the lowest-order terms in $\alpha, \epsilon, \mu$, then the coefficients $a, b$ in $p_{2}(\alpha, \epsilon, \mu)$ are given by

$$
a=\frac{\epsilon^{2}}{32}+\frac{\mu^{2}}{20}, \quad b=\frac{\epsilon^{4}}{64^{2}}+\frac{\mu^{2}}{40}-\frac{\epsilon^{2} \mu^{2}}{320} .
$$

Consequently, up to higher-order terms in $\alpha, \epsilon, \mu$, we have $\lambda=-\left(\epsilon^{2} \mu^{2} / 256\right)<0$, and hence the bifurcation equation (3.5) has two simple zeros. Again, we get that the bifurcation surfaces are given by (3.11).

If $n=4, \mu=0$, the power series expansion of the bifurcation curves $\sigma^{2}=\sigma^{2}(\epsilon)$ has the form

$$
\sigma^{2}(\epsilon)=16+k \epsilon^{3}+\cdots .
$$

The same ideas as above can be applied, but much higher approximations are needed.

\section{REFERENCES}

[1] S. N. Chow, J. Hale, and J. Mallet-Paret, Applications of generic bifurcation, I, Arch. Rat. Mech. Anal. 59, 159-188 (1975)

[2] J. Hale, Oscillations in nonlinear systems, McGraw-Hill, 1963

[3] J. Hale, Ordinary differential equations, Interscience, 1969

[4] L. Rubenfeld, The stability surfaces of a Hill's equation with several small parameters, J. Appl. Mech. 40, $1107-1109$ (1973)

[5] A. Markushewich, Theory of functions of a complex variable, Vol. II, Prentice-Hall, 1965 